\title{
Evaluations of Poly(vinyl alcohol)/Alginate Hydrogels Cross-linked by $\gamma$-ray Irradiation Technique
}

\author{
Sang Yong Nam \\ Department of Polymer Science and Engineering, Engineering Research Institute, Gyeongsang National University, \\ 900 Gazwa-dong, Chinju 660-701, Korea \\ Young Chang Nho \\ Korea Atomic Energy Research Institute, Daejeon 305-600, Korea
}

Seung Hwa Hong

Blood Products Division, Biologics Evaluation Department, Korea Food and Drug Administration, Seoul 122-704, Korea

\begin{abstract}
Gue Tae Chae ${ }^{a}$, Hong Seok Jang ${ }^{b}$, Tae Suk Suh ${ }^{c}$, Woong Shick Ahn, Kyu Eun Ryu, and Heung Jae Chun*
${ }^{a}$ Department of Pathology, ${ }^{b}$ Department of Therapeutic Radiology, ${ }^{c}$ Department of Biomedical Engineering, and Department of Biomedical Sciences, College of Medicine, Catholic University, Seoul 137-701, Korea
\end{abstract}

Received December 5, 2003; Revised February 4, 2004

\begin{abstract}
In this work, we prepared hydrogels for wound dressing from a mixture of poly(vinyl alcohol) (PVA) and alginate using the ${ }^{60} \mathrm{Co} \gamma$-ray irradiation technique. We examined the physical properties of these hydrogels, including gelation, water absorptivity, and gel strength, to evaluate the applicability of these hydrogels for wound dressings. The biocompatibility of these hydrogels was also evaluated in vitro, in cultures of mouse fibroblasts, and in vivo, by subcutaneous implantation studies in rats. The gel content and strength increased upon increasing the radiation dose and upon decreasing the concentration of alginate. The degree of swelling was inversely proportional to the gel content and strength. The degree of cytotoxicity of the $\gamma$-ray-treated hydrogels was ca. $60 \%$ compared to the (-) control (serum) after 1 day of incubation. When the incubations were prolonged up to 2 days, the toxicity of all the samples decreased remarkably and reached that of the control. Subcutaneous implantation studies in rats indicated that foreign body reactions occurring around the implanted hydrogels were moderate and became minimal upon increasing the implantation time.
\end{abstract}

Keywords: biocompatibility, cross-linked hydrogels, cytotoxicity, $\gamma$-ray irradiation, poly(vinyl alcohol), sodium alginate.

\section{Introduction}

Alginates, as naturally derived polysaccharide block copolymers, have been extensively studied for tissue engineering applications as the matrices for cellular encapsulation and culture. ${ }^{1}$ The hydrophilic nature of the alginates enabled the excellent cell loading, and the seeded cells maintained their viability and function in culture. In addition, alginates may provide gelation and moist healing which promotes re-epithelialization. ${ }^{2}$ Therefore, particular attention has been focused on alginates for wound dressing construction. However, alg-

*e-mail: chunhj@ catholic.ac.kr

1598-5032/04/219-06@2004 Polymer Society of Korea inates have been considered limited in their applications for wound dressing materials because of shortage of processing and mechanical properties. ${ }^{3}$ A combination of alginates and synthetic polymers can endow the optimal properties necessary for wound repair.

Poly(vinyl alcohol) (PVA) hydrogel has gained popularity as a scaffold for tissue engineering due to its high water content, good biocompatibility, and consistency similar to soft tissue. ${ }^{4}$ PVA hydrogel consists of 3-dimensional network of polymer chains, which may facilitate diffusional exchange of nutrients and waste products with surrounding environment to allow cell growth. In addition, the mechanical properties of PVA are sufficient to be used as the main component of temporary skin covers or wound dressings. 
So far, chemical methods using small molecular weight cross-linking agents have been widely employed for the preparation of PVA hydrogels. ${ }^{5}$ Unfortunately, these cross-linking processes proved to be cytotoxic. ${ }^{6}$ The irradiation is recognized as a suitable tool for the formation of hydrogels. This process has various advantages, such as the easiness of process control, the possibility of joining hydrogel formation and sterilization in one technological step, and the lack of a need to add any initiators and/or crosslinkers that would be possibly harmful and difficult to remove. ${ }^{3}$ These characteristics make irradiation as the method of choice in the synthesis of hydrogels.

The aim of this work was to evaluate PVA-alginate hydrogel prepared by ${ }^{60} \mathrm{Cobalt}$ gamma-ray $\left({ }^{60} \mathrm{Co} \gamma\right.$-ray) irradiation for use as wound dressing material. The physical properties, including gelation, swelling and gel strength were examined. In addition, as the prerequisites for the clinical usefulness of a skin substitute, the cyto- and tissue compatibility tests of the samples based on the tetrazolium salt (MTT) reduction and subcutaneous implantation were performed.

\section{Experimental}

Materials. PVA (MW: $1.46 \times 10^{3} \sim 8.5 \times 10^{4}$, Aldrich Chemical Company, Milwaukee, WI) and sodium alginate (Kanto Chemical Co. Ltd., Tokyo, Japan) were used without further purification.

Preparation of Hydrogels. Certain amount of PVA was dissolved in double distilled water at $90{ }^{\circ} \mathrm{C}$ and was then mixed with sodium alginate using mechanical stirrer at room temperature to prepare PVA/alginate solution. The dried content of sodium alginate was in the range 1-5 wt $\%$, and the solid concentration of the total PVA/alginate solution was $15 \mathrm{wt} \%$. Each solutions prepared above were loaded into Tygon tube, $15 \mathrm{~cm}$ in length and $1.6 \mathrm{~mm}$ in inner diameter, using hypodermal syringe. Ends of tubes were sealed by stainless steel clip. Then, the tubes containing solutions were exposed to ${ }^{60} \mathrm{Co} \gamma$-ray irradiation of 25,35 , and $50 \mathrm{kGy}$, respectively.

Gel Content. ${ }^{3}$ After irradiation, the crosslinked hydrogel was dried under vacuum for $48 \mathrm{~h}$ at room temperature and weighed. Then the hydrogel was kept in hot distilled water at $60{ }^{\circ} \mathrm{C}$ for $48 \mathrm{~h}$. The insoluble part of hydrogel, which consisted only the crosslinked hydrogel, was dried in vacuum at $60{ }^{\circ} \mathrm{C}$ for $48 \mathrm{~h}$ until the hydrogels reached a constant weight. The gel content was defined as follows:

Gel $(\%)=\left(W_{d} / W_{i}\right) \times 100$, where $W_{d}$ is the dried weight of hydrogel after extraction and $W_{i}$ is the initial weight of the hydrogel.

Degree of Swelling. ${ }^{7}$ The weight of completely dried gels was measured. Then the samples were dipped into the test tubes filled with PBS ( $\mathrm{pH}$ 7.4) whose temperature was maintained at $36^{\circ} \mathrm{C}$ in an incubator. Degree of swelling of these samples was defined as follows:
Degree of swelling $(\%)=\left(W_{s}-W_{d}\right) / W_{d} \times 100$, where $W_{s}$ is the weight of the swollen gels. This measurement had been repeated until the weight of these gels was in fully swollen state.

Tensile Strength and Elongation of Hydrogel. ${ }^{\mathbf{8}}$ Tensile strength under dry and swollen state was measured by Instron (Toyo Baldwin UTM-4-100) at a cross-head speed of $5 \mathrm{~mm} / \mathrm{min}$ and chart speed of $20 \mathrm{~min} / \mathrm{min}$, using $5 \mathrm{~kg}_{\mathrm{f}}$ of load cell.

Cell Culture. The cytotoxicity of hydrogels was evaluated on mouse fibroblasts. L929 fibroblasts (ATCC CCL1, NCTC clone 929, from mouse connective tissue) were cultivated in 10\% FBS-Dulbeccos modified Eagles medium (DMEM, Sigma, St. Louis, MO, USA). The fibroblasts for monolayer cultures were seeded at a density of $5 \times 10^{5}$ cells $/ 2 \mathrm{~mL}$ in six-well culture dishes, and incubated in $5 \% \mathrm{CO}_{2}$ at $37^{\circ} \mathrm{C}$.

Preparation of Extracts. The hydrogels of $1 \mathrm{~g}$ were immersed in $5 \mathrm{~mL}$ serum-supplemented cell culture media. The extracts were prepared by heating for $24 \mathrm{~h}$ in an incubator preferably containing $5 \pm 1 \%$ of carbon oxide and were maintained at $37 \pm 1{ }^{\circ} \mathrm{C}$, because higher temperatures may cause denaturation of serum protein.

MTT Assay. Monolayer culture of L 929 was initiated at a density of $1 \times 10^{3}$ cells per 96 well and incubated for $24 \mathrm{~h}$. Following incubation, the cell culture media were aspirated from the monolayers, and were replaced with serum-supplement cell culture medium extracts. The extracts were tested in duplicate at $25 \%$ of extraction concentration. All cultures were incubated for 24 and $48 \mathrm{~h}$ at $37 \pm 1{ }^{\circ} \mathrm{C}$ in an incubator preferably containing $5 \pm 1 \%$ of carbon dioxide. The amounts of surviving cells after incubation were evaluated by MTT staining assay. In order to perform this test aseptically, hydrogels were washed and rinsed with sterilized PBS pH 7.4, and autoclaved, even though the gels were treated by $\gamma$-ray.

Subcutaneous Implantation Studies. The rod type of hydrogels were cut into strips of $1.5 \mathrm{~mm}$, and inserted into a sterile trocar of $15 \mathrm{G}$ aseptically. SD rats (180 200 g) were anaesthetized with ketamine and rumpun. The hair on the back was shaved, and disinfected with Potadine ${ }^{\circledR}$. Then hydrogels loaded inside the trocars were subcutaneously implanted bilaterally, in parallel with the spine. Two rats were used per time point and per treatment. The animals were sacrificed sequentially at 1, 7, 21, and 35 days after implantation, and the implantation sites were completely excised and processed for classical histology. Biopsy samples were fixed in $4 \%$ neutral formaldehyde solution, and fixed tissues were embedded in paraffin and sectioned at $5 \mu \mathrm{m}$. The histological sections were classically stained with haematoxylin and eosin.

\section{Results and Discussion}

Physical Properties of Hydrogel. Destruction of body 
skin by injuries brings about increase in fluid loss, in turn, produces dehydration state resulting in the loss of the equilibrium of body temperature. By contrast to the above, if the moisture flux trough the skin substitute is excessively retained, fluid, mainly exudates, accumulates at the interface between wound bed and the graft. This accumulated exudates produces facilitated inflammation reaction resulting in procrastination of wound healing process. Consequently, ideal skin graft has to have the proper swelling characteristics to absorb large amount of exudates to minimize the edema formation at the interface.

Hydrogels have gained popularity as wound dressing materials due to their high water content with proper water vapor transmission rate. Figure 1 shows the gelation and degree of swelling behavior of the hydrogels prepared by $\gamma$ ray irradiation. The gel content was in the range of $75-95 \%$. The gel content increased with decrease in the amount of alginate in PVA/alginate hydrogels. This may be attributed to the differences in dissolution characteristics of two polymers. However, the increase in the radiation dose was found to surmount this problem to a certain extent. The degree of swelling was in the range $600-950 \%$. The degree of swelling increased as concentration of alginate increased and as the radiation dose decreased. The swelling percentage was inversely proportional to the gel percentage.

The durability and the elasticity are also important characteristics of the ideal skin substitute as the barrier of the body. Dressing materials having $100 \mathrm{~kg}_{\mathrm{f}} / \mathrm{cm}^{2}$ in tensile strength and $20 \%$ in elongation have been favored to graft. ${ }^{19,20}$ The tensile strength and elongation of hydrogels under dry and wet condition are shown in Figures 2 and 3, respectively. The tensile strength of the PVA/alginate hydrogels increased as the concentration of alginate decreased and as the radiation dose increased. This may imply the fact that brittle

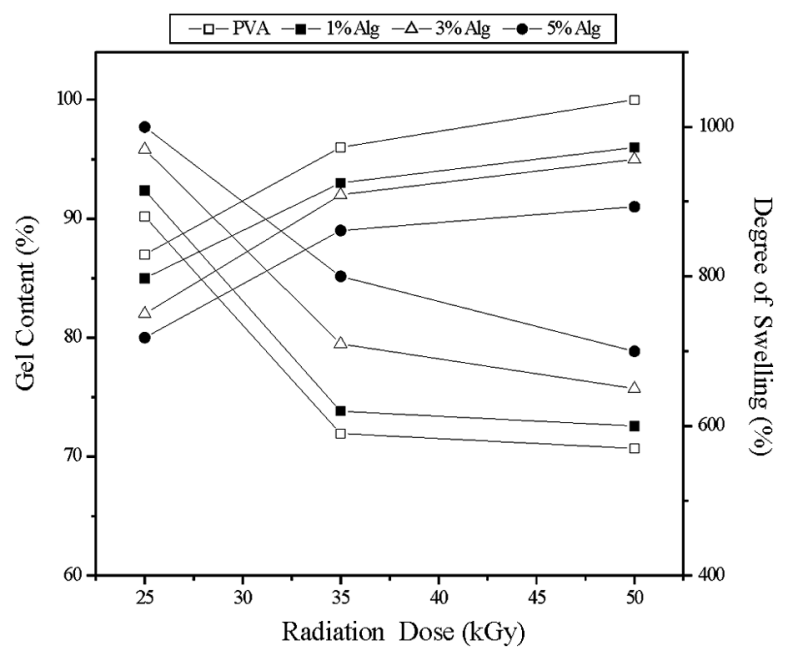

Figure 1. Gel contents and degree of swelling of PVA/alginate hydrogels as a function of irradiation dose (left: means \pm 3.9, right: means $\pm 4.8, \mathrm{n}=3$ ). characteristics of alginate ${ }^{2}$ at dry state can be compensated by the toughness of PVA. As compared to dry condition, the tensile strength of all hydrogels under swollen state showed a trend of decrease. Nevertheless, all of the swollen samples have tensile strength and elongation over $150 \mathrm{~kg}_{\mathrm{f}} / \mathrm{cm}^{2}$ and $70 \%$, respectively. Therefore, the samples could be considered as stable and durable skin substitute when they were soaked by fluid. ${ }^{19,20}$

In vitro Cytotoxicity Test. Cytotoxicity of hydrogels crosslinked by $\gamma$-ray irradiation was evaluated on mouse fibroblast cultures. Figure 4 shows the MTT reduction in mouse fibroblast cultures after 1 and 2 days of incubation, in the presence of extracts of test samples. After 1 day of incubation, the MTT reduction capacity of $\gamma$-ray crosslinked hydrogels was found to be $\sim 60 \%$. This can be ascribed to

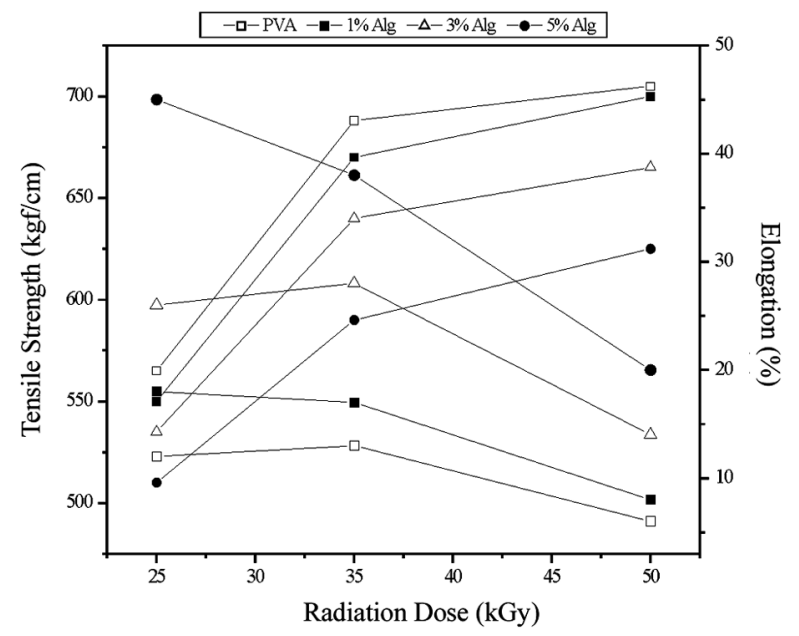

Figure 2. Tensile strength and elongation of gels at completely dried state (left: means \pm 36 , right: means $\pm 4.8, \mathrm{n}=3$ ).

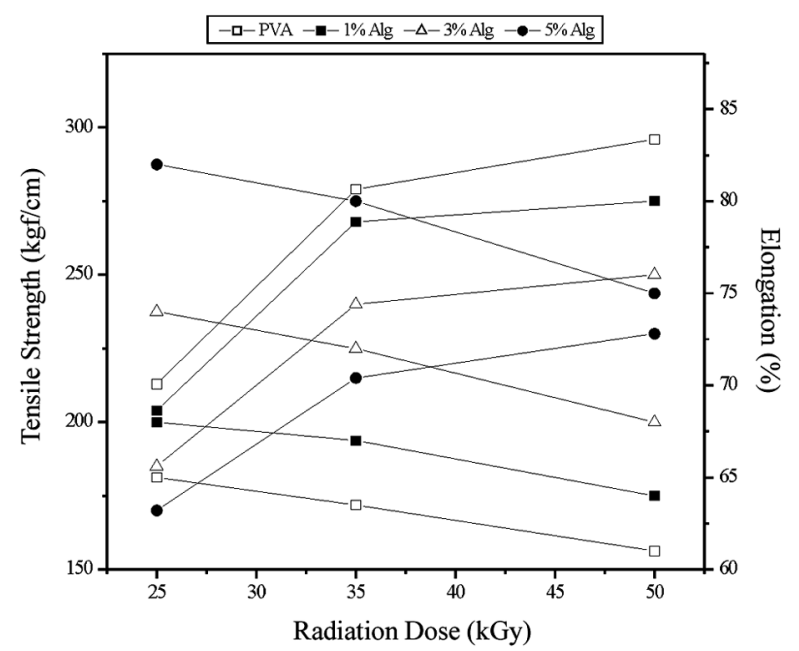

Figure 3. Tensile strength and elongation of gels at fully swollen state (left: means \pm 15.3 , right: means $\pm 3.7, n=3$ ). 


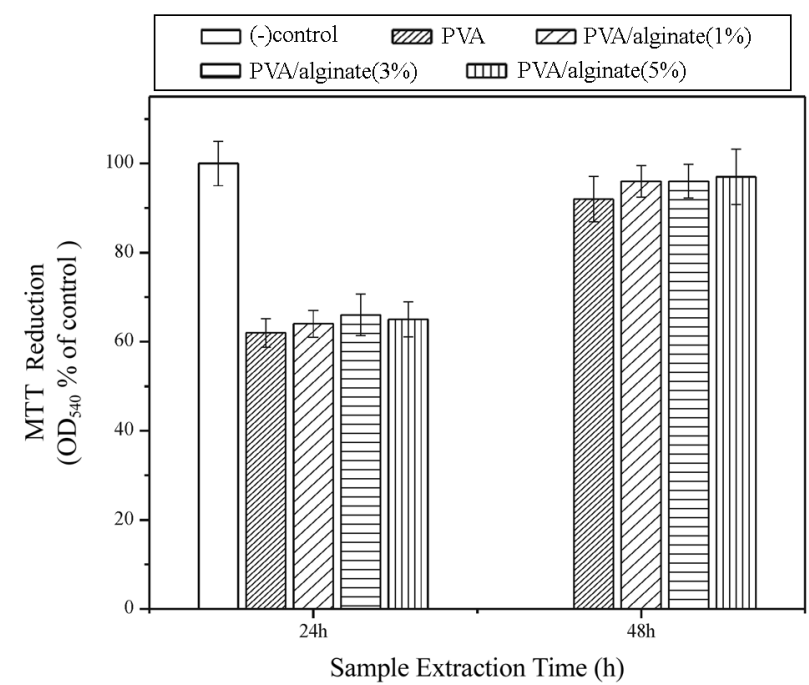

Figure 4. MTT reduction capacity expressed as the percentage of control $(n=3)$.

presence of the residual degraded products in serum-supplement cell culture medium. When the incubations were prolonged up to 2 days, however, the toxicity of all samples decreased dramatically. This represents that the cytotoxicity of degraded products of hydrogels due to $\gamma$-ray irradiation was almost negligible.

Subcutaneous Implantation Studies. Alginates are known to facilitate the management of highly exudating lesions due to its great hydration capacity with low rate of degradation and toxicity. ${ }^{18}$ Therefore, the addition of alginate to PVA hydrogel may produce the improved tissue compatibility. To evaluate this performance, the changes in acute and chronic foreign body reactions between PVA and PVA/alginate hydrogels were determined by subcutaneous implantation in rats.

At day 1 after implantation, PVA hydrogel was covered by a large number of acute cells, mainly consist of polymorphonuclear leukocytes and monocytes (Figure 5(A)). The surrounding tissue exhibited some degree of edema and congestion (Figure 5(B)). Figure 6(A), (B) and (C) shows

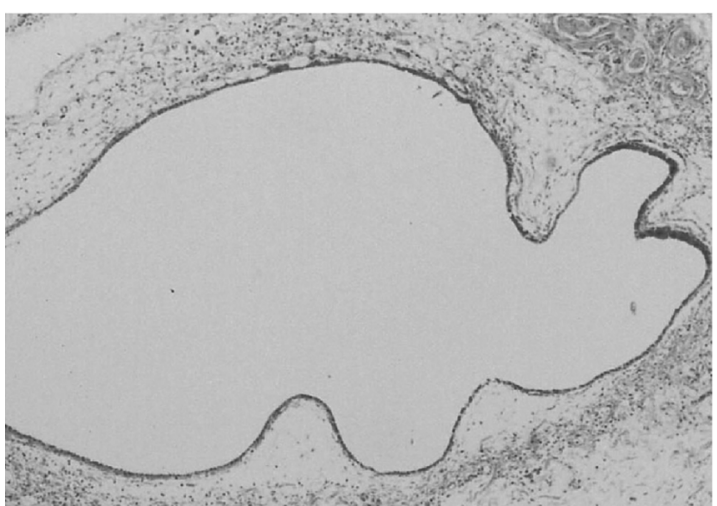

(A)

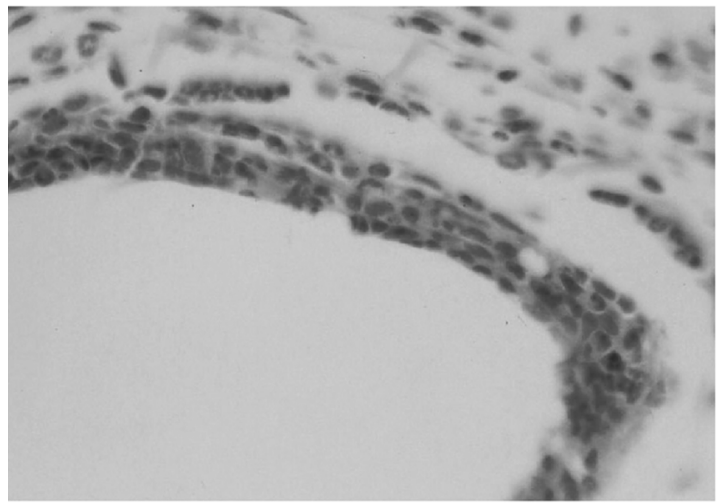

(B)

Figure 5. (A) Acute inflammatory cells, polys and monocytes, and edema surrounded PVA injected underneath the muscle layer, 1 day, magnification $10 \times 4$ (ocular lens $\mathrm{x}$ objective lens); (B) Higher magnification of (A) presented band of acute inflammatory cells and edema walled off PVA in the skin, 1 day, $10 \times 20, \mathrm{H} \& \mathrm{E}$.

the late changes in foreign body reactions against PVA implants. The PVA hydrogel was surrounded by fibroblast capsule that consists of monocytes and infiltrated macrophages with small number of foreign body giant cells (Figure 6(A)). A large number of neo-vasculatures, capillaries and venules, as well as fibrosis granulation tissues were also

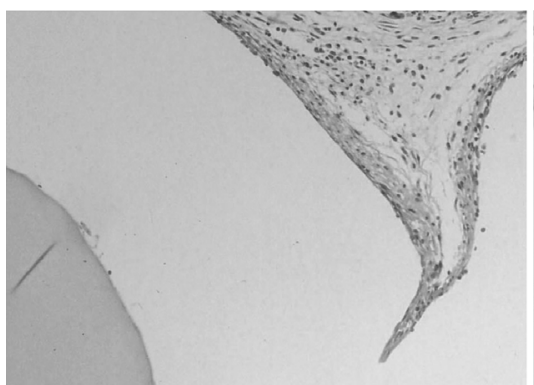

(A)

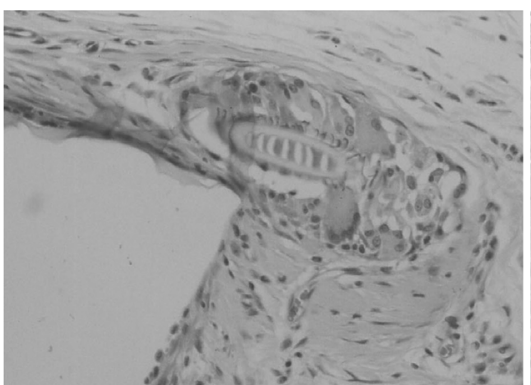

(B)

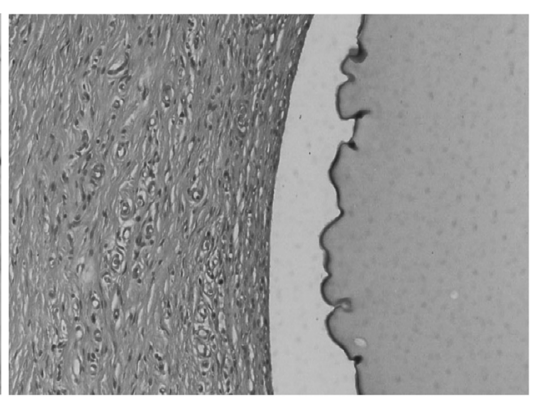

(C)

Figure 6. (A) Granulation tissue, newly growing capillaries and fibroblasts, grew into the foreign material, PVA group, 1 week, $10 \times 10$; (B) Foreign body giant cells were found in the wall of granulation tissue, PVA group, 3 weeks, 10× 20; (C) Thick band of fibrosis walled off the injected PVA material at the injected site of the skin, PVA group, 5 weeks implantation, $10 \times 10, \mathrm{H} \& \mathrm{E}$. 
observed at the interface. Granulation tissues started decreasing after 3 weeks of implantation (Figure 6(B)) and disappeared at 5 weeks after implantation (Figure 6(C)). Fibrous connections extend to the capsule of the distal radius, and this capsule appears to compress the neighboring tissue at this period.

PVA/alginate sample (5\% of alginate) showed mild foreign body reactions with some degree of compression necrosis due to mechanical pressure produced by the swelling of implants (Figure 7(A)). Infiltrated chronic cells as well as giant cells were found at the interface of PVA/alginate samples (Figure 7(B)), but these chronic reactions were almost disappeared from 3 to 5 weeks of implantation (Figure 7(C) and (D)). In addition, this sample showed very low degree of fibrosis; the fibroblasts were barely found.

Implantation of a biomaterial is always associated with an acute inflammatory response followed by a chronic inflammation. Both processes are of some duration (days to months depending materials), which might induce the infiltration of polymorphonuclear leukocytes (PMNs), macrophages, fibroblasts, and some lymphocytes as well as formation of fibrin, exudates and new blood vessels. ${ }^{9}$ End of this paper showed theses reactions against both PVA and PVA/alginate hydrogels cross-linked by $\gamma$-ray irradiation technique.

Acute inflammation is of relatively short duration, lasting from few minutes to days. Its main characteristics are the exudation of fluid and plasma proteins (edema) and the emigration of leukocytes (predominantly PMNs). ${ }^{9}$ Although the nature of the initial tissue response during the first 7 days was similar for both PVA and PVA/alginate hydrogel samples used in this study, the amount of exudates and the number of acute cells were different. PVA, exhibited decreased extent of acute cell reactions compared to partly biodegradable PVA/alginate hydrogel. This indicates that alternations of acute foreign body reactions were specifically related to biodegradation of the materials. The acute inflammatory response to biomaterials, as reviewed by Tang et al., is triggered by the surrounding host proteins such as IgG and complement. ${ }^{10}$ These proteins are known to adsorb to biomaterials, and neutrophils and macrophages have corresponding cell membrane receptors for these opsonins. These receptors may also play a role in the activation of the

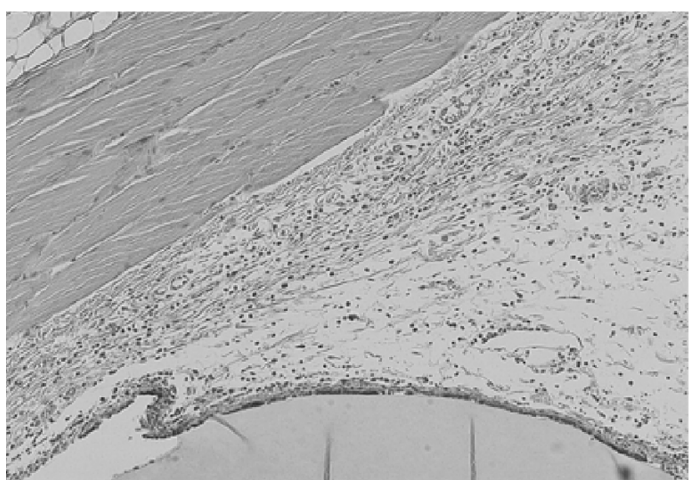

(A)

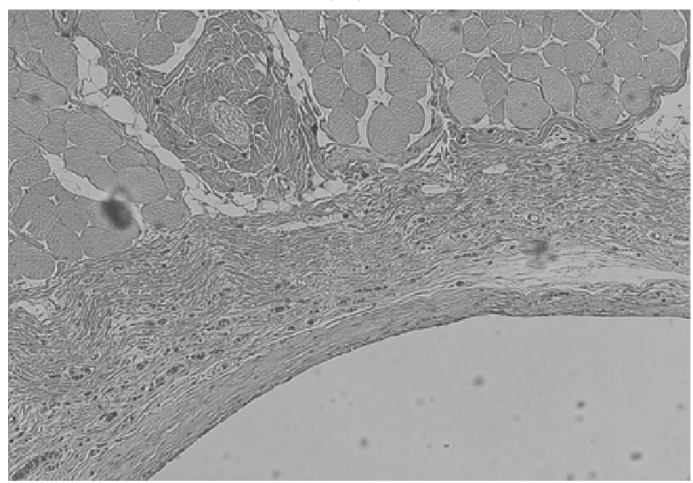

(C)

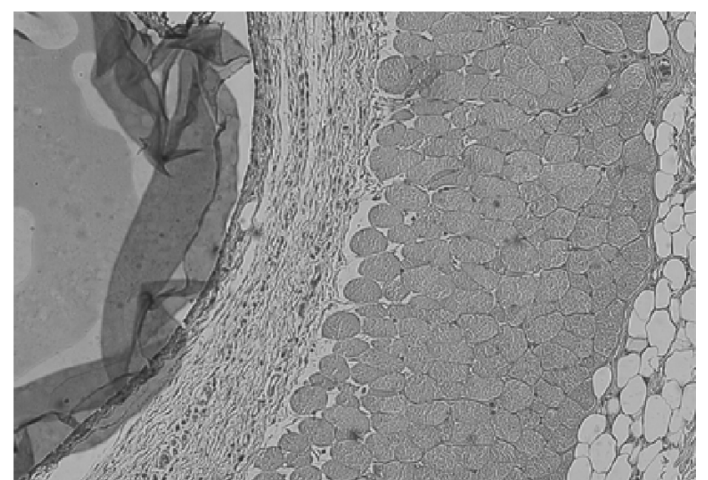

(B)

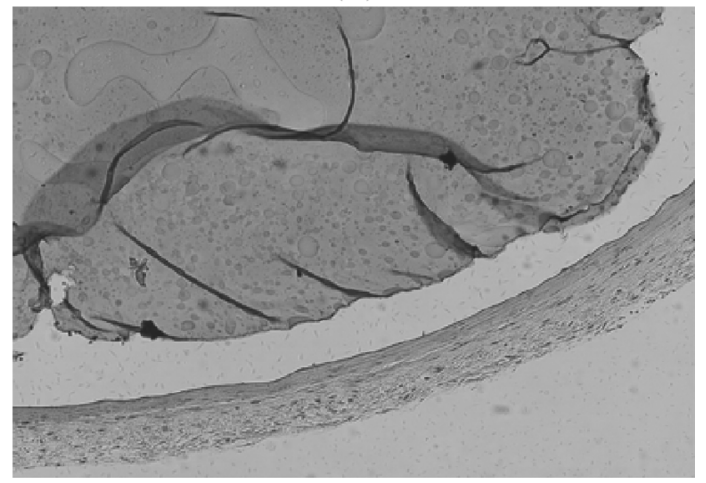

(D)

Figure 7. Foreign body reactions of PVA/ 5\% alginate hydrogel (A) at 1 day of implantation; this gel showed enhanced absorption of exudates with large population of PMNs. The swelling of this gel triggered compression necrosis of muscle cells to some extent, (B) at 1 week of implantation; thin layer of fibroblasts was found at the interface with some extent of eosinophils and giant cells. The cells in the muscle layer started to have their original shape, $(\mathrm{C})$ at 3 weeks of implantation; foreign body giant cells and a few of eosinophils were found in the wall, (D) at 5 weeks of implantation; Number of giant cells and the fibrosis was remarkably decreased. Chronic inflammatory Response was almost absent at the interface, $10 \times 10, \mathrm{H} \& \mathrm{E}$. 
attached neutrophil or macrophage. ${ }^{11,12}$ For non-degradable polymers, owing to disparity in size between biomaterial surface and the attached cell, frustrated phagocytosis may occur. ${ }^{13}$ For biodegradable materials, PMNs may release enzymes by direct extrusion or exocytosis, and the amount of enzyme during this process depends on the size of polymer particle, with larger particles inducing greater amounts of enzyme release, resulting in the present of larger populations of acute cells at the interfaces of biodegradable hydrogels than non-degradable samples. ${ }^{13}$

The amount of exudates formation against the implants largely depends on the hydration capacity of the implant. M. J. Waring et al. suggested that the materials with high hydration properties could be beneficial in the treatment of edema by absorption of exudative fluids. ${ }^{14}$ As shown in Figure 1, the swell degree of PVA/alginate gels increased with increase in the content of alginate. The increased content of alginate produced enhanced adsorption of exudates, in turn, showed the decreased degrees of edema formation at the interface, ${ }^{15}$ though a little degrees of compression necrosis were found. On the other hand, PVA exhibited increased edema formation with great numbers of neo-vascular congestions due to decreased water uptake property.

The chronic inflammation due to the implantation of biomaterials can be characterized by decreased numbers of PMNs and increased numbers of macrophages, lymphocytes, giant cells, and fibroblasts which are actively involved in repair reaction by forming fibrous tissue. ${ }^{12}$ After 3 weeks of implantation, non-degradable sample, PVA hydrogel, exhibited above-mentioned typical chronic inflammatory responses including final phase tissue reactions characterized by a thick fibrous capsule with small numbers of macrophages. Tissue response to polymeric materials accompanying biodegradation can be categorized into several types, depending on the degree of toxicity, stimulated effect, diffusion of degraded products, and the rate and duration of degradation. ${ }^{16}$ The polymer producing stimulating substances with high rate of degradation will provoke a severe acute and chronic inflammation. On the other hands, the polymer producing low toxic degraded substances persistently may cause a mild chronic inflammation that exists as long as degradation continues. Alginate, as studied by Ueyama, the rate of degradation and the degree of toxicity is very low, which enables that the inflammatory response was almost absent surrounding alginate samples. ${ }^{17}$

\section{Conclusions}

In this work, PVA/alginate hydrogels were prepared by ${ }^{60} \mathrm{Co} \gamma$-ray irradiation technique. The gel content and gel strength increased as the concentration of alginate decreased and as radiation dose increased. The swelling degree of hydrogels increased as the concentration of alginate increased and as radiation dose decreased. The subcutaneous implan- tation studies revealed that initial foreign body reaction to non-degradable hydrogels was mild compared to degradable hydrogels. At days 21 and 35 post-implantation, nondegradable hydrogels showed typical chronic inflammatory responses including final phase tissue reactions characterized by a thick fibrous capsule with small numbers of macrophages. On the other hand, PVA/alginate hydrogels, for longterm implantation, showed the decreased foreign body reactions with mild fibrosis compared to PVA hydrogel, which could be dependant upon low toxicity of degradation products and prolonged rate and duration of degradation of alginate. These results demonstrate that $\gamma$-ray cross-linked hydrogels possessing alginate can be considered biocompatible and therefore are promising materials for wound healing purpose. Presently, we are examining the healing effect of alginatebased skin substitutes in vivo.

Acknowledgements. This work has been supported by Korea Food and Drug Administration; Grant, KFDA-03091TIS-042.

\section{References}

(1) M. M. Stevebs, H. F. Qanadilo, R. Langer, and V. P. Shastri, Biomaterials, 25, 887 (2004).

(2) J. V. Edwards, A. F. Bopp, S. L. Batiste, and W. R. Goynes, J. Biomed. Mater. Res., 66A, 433 (2003).

(3) Y. C. Nho and K. R. Park, J. Appl. Polym. Sci., 85, 1787 (2002).

(4) R. H. Schmedlen, K. S. Masters, and J. L. West, Biomaterials, 22, 4325 (2002).

(5) Y. Tabata and Y. Ikada, Biomaterials, 20, 2169 (1999).

(6) J. P. Draye and B. Delaey, A.Van de Voorde, A. Van Den Bulcke, B. De Reu, and E. Schacht, Biomaterials, 19, 1677 (1998).

(7) S. H. Yuk and S. H. Cho, Korea Polym. J., 8, 89 (2000).

(8) J. W. Park, D. J. Lee, E. S. Yoo, S. S. Im, S. H. Kim, and Y. H. Kim, Korea Polym. J., 7, 93 (1999).

(9) J. M. Anderson, ASAIO, 11, 101 (1988).

(10) L. Tang and J. W. Eaton, J. Exp. Med., 178, 2147(1993).

(11) W. J. Maloney, D. H. Sun, Y. Nakashima, R. James, and R. L. Smith, J. Biomed. Mater. Res., 41, 371 (1998).

(12) T. Rae, Crit. Rev. Biocompatibility, 2, 97 (1986).

(13) P. M. Henson, J. Immunol., 107, 1547 (1971).

(14) M. J. Waring and D. Parsons, Biomaterials, 22, 903 (2001).

(15) Y. Suzuki, Y. Nishimura, M. Tanihara, K. Suzuki, T. Nakamura, Y. Shimizu, Y. Yamawaki, and Y. Kakimaru, $J$. Biomed. Mater. Res., 39, 317 (1998).

(16) Y. Imai and A. Watanabe, Jpn. J. Artif. Organs, 15, 252 (1986).

(17) Y. Ueyama, K. Ishikawa, T. Mano, T. Koyama, H. Nagatsuka, K. Suzuki, and K. Ryoke, Biomaterials, 23, 2027 (2002).

(18) M. Limova, Ostomy Wound Manage., 29, 26 (2003).

(19) P. M. Neumann, B. Zur, and Y. Ehrenreich, J. Biomed. Mater. Res., 15, 9 (1981).

(20) I. V. Yannas and J. F. Burke, J. Biomed. Mater. Res., 14, 65 (1980). 